\title{
Multi-dimensional Characteristics Analysis of Decathlon Champion Achievement of Modern Olympic Game
}

\author{
Changmei Huang \\ Institute of Physical Education \\ Hunan University of Science and Technology \\ Xiangtan Hunan, P.R. China \\ changmeihuang@126.com
}

\author{
Weihua Shen \\ Institute of Physical Education \\ Hunan University of Science and Technology \\ Xiangtan Hunan, P.R. China \\ whshen2007@yahoo.com.con
}

\begin{abstract}
Using the methods of literature material, expert interview, quantity statistics and grey correlation analysis, the paper has carried on the multiple perspectives and multi-level grey correlation analysis of Olympic men's decathlon champion achievement. The results reveal the relationship of total result and events; relationship of events and individual event, and then, gets the further discussion and analysis, relevant conclusions. The result aimed to establish scientific training plan for decathlon coaches, and prepare for the next Olympic game with the beneficial reference and strategic significance.
\end{abstract}

Keywords-Decathlon; Olympic Game; champion result; Grey relational analysis

\section{INTRODUCTION}

Grey correlation analysis of the grey system theory is a quantization process which studies the close degree of each factor against the main factors, and that is the comparison of the quantitative and analysis of relevant factors (sub-factors and main factors) The grey relational analysis theory is a powerful algorithm tool which can solve the question in which partial information is vague and unclear. Because there are many events in the Olympic games' track and field, and decathlon achievement is influenced by those factors, such as athletes individual growth cycle, the physical quality, the outstanding performance of some athletes, and injuring and retirement of outstanding athletes, the using of high tech sports training instrument, the implementation plan of scientific training guidance, the introduction of advanced technology, facilities and improvement. Those can make the match result uncertain, and the decathlon achievement has obviously grey features, and so, we can view decathlon achievement system as a grey system. In this paper, based on the classification of professor Tian MaiJiu in 1993, all events can be classified into velocity $(100$ m, 400 m, 110 m hurdles), power(long jump and throw), skip(high jump, pole vault, jump), throwing(shot put, discus, javelin), and endurance(1500 meters). The paper uses grey relational analysis method to compute the grey correlation degree of modern Olympic men's decathlon champion results, to study the influence degree of each group between the total score and each individual event in order to reveal dependencies relation between events, and to improve selecting of the world elite male decathlon and making training plan with beneficial reference.

\section{RESEARCH OBJECTS AND METHODS}

\section{A. Research Objects}

The paper collects decathlon champion performance of Olympic Games. According to the international association of athletics federations formulation method in 1985, performances are divided into seven stages, that is 1920-1928(from the 7th to the 9th: 5881 points, 6561 points, 6561 points); $1932-1948$ (from the 10th to the 14th: 6815 points, 7341 points, 7341 points); 1952-1960 (from the 15 th to 17 th, 7592 points, 7614 points, 7614 points); 1964-1972 (from the 18th to 20th: the 7816 points, 8144 points, 8144 points); 1976-1984 (from the 21st to April 23, 8634, 8522 points, 8522 points); 1988-1996 (from the 24th to April 26:8488 points, 8611 points, 8611 points); 20002012 (from the 27th to 30th, 8641 points, 8893 points, 8791 points, 8893 points)..

\section{B. Research Methods}

The paper collects 100 research papers of recent domestic and foreign literatures related to men's decathlon sports, and collects achievements of the Olympic men's decathlon champion in the race. Then, we use mathematical statistics, statistical software EXCEL to process those data. At last, we use grey relation analysis method of grey system theory to compute the degree, sequence and weight of the modern Olympic men's decathlon champion result in multiple perspectives and multi-level grey correlation analysis.

\section{PROCESS OF GREY CORRELATION ANALYSIS}

Procedure of grey relational analysis is treated as follow:

(1) Determine the factors listed

Mother factor $\mathrm{w}_{0}$ (total result), which is evaluated as follows:

$w_{0}=\left[w_{0}(k)\right], k \in\{1, \cdots, \mathrm{n}\}$, where $\mathrm{n}$ is the number of cases;

Sub-factors $\mathrm{w}_{1}, \mathrm{w}_{2}, \mathrm{w}_{3}, \mathrm{w}_{4}, \mathrm{w}_{5}, \mathrm{w}_{6}, \mathrm{w}_{7}, \mathrm{w}_{8}, \mathrm{w}_{9}, \mathrm{w}_{10}$ (individual results or event-groups), which are evaluated as follow: 
$w_{i}=\left[w_{i}(k)\right], i \in\{1, \cdots, \mathrm{m}\}, k \in\{1, \cdots, \mathrm{m}\}$, where $\mathrm{m}$ is number of individual or event-group.

(2) Determine the spaces of factors @GRF.

@GRF is evaluated as follow:

$$
\begin{gathered}
x_{i}=A V G w_{i}, A V G: x_{i}(k)=\frac{w_{i}(k)}{w_{i}(\operatorname{avg})} \\
w_{i}(\operatorname{avg})=\frac{1}{n} \sum_{k=1}^{\mathrm{n}} w_{i}(k), i \in\{0, \cdots, \mathrm{m}\} .
\end{gathered}
$$

(3) Determine $\triangle \mathrm{GR}$

The smallest difference and the polarization the polarization maximum differential, which is evaluated as follows:

$$
\Delta_{0 i}(k)=\left|x_{0}(k)-x_{i}(k)\right|, i \in\{1, \cdots, \mathrm{m}\}, k \in\{1, \cdots, \mathrm{m}\},
$$

(4) Seek correlation (first order correlation coefficient then order correlation)

By $\triangle_{\mathrm{GR}}$, we can get $\triangle_{0 \mathrm{i}}$ (max) and $\triangle_{0 \mathrm{i}}$ (min), make the identification coefficient, $\xi=0.5$, and grey correlation coefficient

$$
\begin{gathered}
\left.\Delta_{0 i}(k)=\left|x_{0}(k)-x_{i}(k)\right|\right\} \\
i \in\{1, \cdots, \mathrm{m}\}, k \in\{1, \cdots, \mathrm{n}\}, \\
r\left(x_{0}(k), x_{i}(k)\right)=\frac{\Delta_{0 i}(\min )+\xi \Delta_{0 i}(\max )}{\Delta_{0 i}(k)+\xi \Delta_{0 i}(\max )} .
\end{gathered}
$$

(5) Seek grey relational grade, sequence, and weight

$$
r\left(x_{0}, x_{k}\right)=\frac{1}{n} \sum_{k=1}^{\mathrm{n}} r\left(x_{0}(k), x_{i}(k)\right), w_{i}=r_{i} / \sum r_{k} \text {. }
$$

\section{RESUlTS AND DISCUSSION}

\section{A. Grey Correlation Analysis of Total Result against Event-groups}

According to the theory of professor Tian Maijiu, ten events can be divided into four classes, that are speed event-group (100m, 400m, 110m hurdles), jump eventgroup(high jump, long jump and pole vault), throwing event-group(shot put, discus, javelin) and endurance event-group $(1500 \mathrm{~m})$. According to grey correlation analysis formula, we can get the results shown in table 1 .

From table 1: (1)The grey relational sequence of four event-groups against total in the 7th to 9th stage is endurance event-group $>$ speed event-group $>$ skip eventgroup $>$ throwing event-group, and their weight sequence is the same as the grey relational sequence. From the grey relational degree and weight, the biggest contribution to total score is endurance event-group, accounted for $27.40 \%$, followed by the speed event-group, accounted for $26.63 \%$, and the smallest contribution is throwing eventgroup, accounted for $22.30 \%$. All those show that endurance event-group has the biggest influence on the total score, also reflect their training level of endurance is relatively high, and while throwing event-group is relatively weak.
(2)The grey relational sequence of four event-groups against total in the 10th to 14th stage is skip eventgroup $>$ speed event-group $>$ throwing eventgroup>endurance event-group, and their weight sequence is the same as the grey relational sequence. From the grey relational degree and weight, the biggest contribution to total score is skip event-group, accounted for $31.39 \%$, followed by the speed event-group, accounted for $25.99 \%$, and the smallest contribution is endurance event-group, accounted for $17.69 \%$. All those show that skip eventgroup has the biggest influence on the total score, also reflect their training level of skip event-group is relatively high, while endurance event-group is relatively weak.

TABLE I. GREY CoRrelation DEgREE, SEQUENCE, WEIGHT AND WeIght SEQUENCE Of TOTAL AND EVENT-GROUPS IN DIFFERENT STAGES

\begin{tabular}{|c|c|c|c|c|c|}
\hline Period & References & Speed & Skip & Throwing & Endurance \\
\hline \multirow{3}{*}{$7-9$} & GRD & 0.6057 & 0.5384 & 0.5072 & 0.6232 \\
\cline { 2 - 6 } & GRS & 2 & 3 & 4 & 1 \\
\cline { 2 - 6 } & W(\%) & $26.63 \%$ & $23.67 \%$ & $22.30 \%$ & $27.40 \%$ \\
\hline \multirow{3}{*}{$10-14$} & GRD & 0.6775 & 0.8183 & 0.6501 & 0.4613 \\
\cline { 2 - 6 } & GRS & 2 & 1 & 3 & 4 \\
\cline { 2 - 6 } & W(\%) & $25.99 \%$ & $31.39 \%$ & $24.93 \%$ & $17.69 \%$ \\
\hline \multirow{3}{*}{$15-17$} & GRD & 0.4606 & 0.6231 & 0.5713 & 0.7658 \\
\cline { 2 - 6 } & GRS & 4 & 2 & 3 & 1 \\
\cline { 2 - 6 } $18-20$ & W(\%) & $19.03 \%$ & $25.74 \%$ & $23.60 \%$ & $31.64 \%$ \\
\cline { 2 - 6 } & GRD & 0.7034 & 0.6757 & 0.7863 & 0.4602 \\
\cline { 2 - 6 } & GRS & 2 & 3 & 1 & 4 \\
\hline \multirow{3}{*}{$21-23$} & GRD & $26.79 \%$ & $25.74 \%$ & $29.95 \%$ & $17.53 \%$ \\
\cline { 2 - 6 } & GRS & 0.7991 & 0.7057 & 0.7222 & 0.4595 \\
\cline { 2 - 6 } & W(\%) & $29.74 \%$ & $26.27 \%$ & $26.88 \%$ & $17.10 \%$ \\
\hline \multirow{3}{*}{$24-26$} & GRD & 0.6303 & 0.7216 & 0.6451 & 0.4600 \\
\cline { 2 - 6 } & GRS & 3 & 1 & 2 & 4 \\
\cline { 2 - 6 } & W(\%) & $25.65 \%$ & $29.37 \%$ & $26.25 \%$ & $18.72 \%$ \\
\hline \multirow{3}{*}{$27-30$} & GRD & 0.8345 & 0.8628 & 0.6412 & 0.5826 \\
\cline { 2 - 6 } & GRS & 2 & 1 & 3 & 4 \\
\cline { 2 - 6 } & W(\%) & $28.57 \%$ & $29.54 \%$ & $21.95 \%$ & $19.94 \%$ \\
\hline
\end{tabular}

(3)The grey relational sequence of four event-groups against total in the 15th to 17th stage is endurance eventgroup>skip event-group>throwing event-group>speed event-group, and their weight sequence is the same as the grey relational sequence. From the grey relational degree and weight, the biggest contribution to total score is endurance event-group, accounted for $32.64 \%$, followed by the skip event-group, accounted for $25.74 \%$, and the smallest contribution is speed event-group, accounted for $19.03 \%$. All those show that endurance event-group has the biggest influence on the total score, also reflect their training level of endurance event-group is relatively high, while speed event-group is relatively weak.

(4)The grey relational sequence of four event-groups against total in the 18th to 20th stage is throwing eventgroup>speed event-group>skip event-group>endurance event-group, and their weight sequence is the same as the grey relational sequence. From the grey relational degree and weight, the biggest contribution to total score is throwing event-group, accounted for $29.95 \%$, followed by the speed event-group, accounted for $26.79 \%$, and the smallest contribution is endurance event-group, accounted for $17.53 \%$. All those show that throwing event-group has 
the biggest influence on the total score, also reflect their training level of throwing event-group is relatively high, while endurance event-group is relatively weak.

(5)The grey relational sequence of four event-groups against total in the 21th to 23th stage is speed eventgroup $>$ throwing event-group $>$ skip eventgroup>endurance event-group, and their weight sequence is the same as the grey relational sequence. From the grey relational degree and weight, the biggest contribution to total score is speed event-group, accounted for $29.74 \%$, followed by the throwing event-group, accounted for $26.88 \%$, and the smallest contribution is endurance eventgroup, accounted for $17.10 \%$. All those show that speed event-group has the biggest influence on the total score, also reflect their training level of speed event-group is relatively high, while endurance event-group is relatively weak.

(6)The grey relational sequence of four event-groups against total in the 24th to 26th stage is skip event-group> throwing event-group>speed event-group >endurance event-group, and their weight sequence is the same as the grey relational sequence. From the grey relational degree and weight, the biggest contribution to total score is skip event-group, accounted for $29.37 \%$, followed by the throwing event-group, accounted for $26.25 \%$, and the smallest contribution is endurance event-group, accounted for $18.72 \%$. All those show that skip event-group has the biggest influence on the total score, also reflect their training level of skip event-group is relatively high, while endurance event-group is relatively weak.

(7)The grey relational sequence of four event-groups against total in the 27th to 30th stage is skip event-group> speed event-group $>$ throwing event-group $>$ endurance event-group, and their weight sequence is the same as the grey relational sequence. From the grey relational degree and weight, the biggest contribution to total score is skip event-group, accounted for $29.54 \%$, followed by the speed event-group, accounted for $28.57 \%$, and the smallest contribution is endurance event-group, accounted for $19.94 \%$. All those show that skip event-group has the biggest influence on the total score, also reflect their training level of skip event-group is relatively high, while endurance event-group is relatively weak.

\section{B. Gre Correlation Analysis of Individual Event against Event-group}

From table II, (1)The grey relational sequence of individual event against speed event-group in the 7th to 9th stage is $400 m>100 m>110 m$ hurdles. From the grey relational sequence and weight, the biggest contribution to speed event-group is $400 \mathrm{~m}$, which reflects their training level of $400 \mathrm{~m}$ is relatively high, while $110 \mathrm{~m}$ hurdles is relatively weak.

(2)The grey relational sequence of individual event against speed event-group in the 10th to 14th stage is $110 \mathrm{~m}$ hurdles $>400 \mathrm{~m}>100 \mathrm{~m}$. From the grey relational sequence and weight, the biggest contribution to speed event-group is $110 \mathrm{~m}$ hurdles, which reflects their training level of $110 \mathrm{~m}$ hurdles is relatively high, while $100 \mathrm{~m}$ hurdles is relatively weak.

(3)The grey relational sequence of individual event against speed event-group in the 15th to 17th stage is $100 \mathrm{~m}>110 \mathrm{~m}$ hurdles $>400 \mathrm{~m}$. From the grey relational sequence and weight, the biggest contribution to speed event-group is $100 \mathrm{~m}$, which reflects their training level of $100 \mathrm{~m}$ is relatively high, while $400 \mathrm{~m}$ is relatively weak.

TABLE II. GREY CORRELATION DEGREE, RELATIONAL SEQUENCE, WEIGHT AND WEIGHT SEQUENCE OF SPEED EVENT-GROUP AND INDIVIDUAL EVENT IN DIFFERENT STAGES

\begin{tabular}{|c|c|c|c|c|}
\hline Period & References & $\mathbf{1 0 0 m}$ & $\mathbf{4 0 0 m}$ & $\mathbf{1 1 0 m}$ hurdles \\
\hline \multirow{3}{*}{$7-9$} & GRD & 0.4746 & 0.5099 & 0.4719 \\
\cline { 2 - 5 } & GRS & 2 & 1 & 3 \\
\cline { 2 - 5 } & W(\%) & $32.59 \%$ & $35.01 \%$ & $32.40 \%$ \\
\hline \multirow{3}{*}{$10-14$} & GRD & 0.4636 & 0.4962 & 0.6119 \\
\cline { 2 - 5 } & GRS & 3 & 2 & 1 \\
\cline { 2 - 5 } & W(\%) & $29.50 \%$ & $31.57 \%$ & $38.93 \%$ \\
\hline \multirow{3}{*}{$15-17$} & GRD & 0.7362 & 0.4610 & 0.4685 \\
\cline { 2 - 5 } & GRS & 1 & 3 & 2 \\
\cline { 2 - 5 } & W(\%) & $44.20 \%$ & $27.68 \%$ & $28.13 \%$ \\
\cline { 2 - 5 } & GRD & 0.5831 & 0.6068 & 0.5125 \\
\cline { 2 - 5 } & GRS & 2 & 1 & 3 \\
\hline \multirow{3}{*}{$21-23$} & W(\%) & $34.25 \%$ & $35.64 \%$ & $30.10 \%$ \\
\cline { 2 - 5 } & GRD & 0.4808 & 0.4646 & 0.6939 \\
\cline { 2 - 5 } & GRS & 2 & 3 & 1 \\
\hline \multirow{3}{*}{$24-26$} & G(\%) & $29.33 \%$ & $28.34 \%$ & $42.33 \%$ \\
\cline { 2 - 5 } & GRD & 0.6795 & 0.5333 & 0.4889 \\
\cline { 2 - 5 } & W(\%) & $39.93 \%$ & $31.34 \%$ & $28.73 \%$ \\
\hline \multirow{3}{*}{$27-30$} & GRD & 0.6531 & 0.6083 & 0.6148 \\
\cline { 2 - 5 } & GRS & 1 & 2 & 3 \\
\cline { 2 - 5 } & W(\%) & $34.81 \%$ & $32.42 \%$ & $32.77 \%$ \\
\hline
\end{tabular}

(4)The grey relational sequence of individual event against speed event-group in the 18th to 20th stage is $400 \mathrm{~m}>100 \mathrm{~m}>110 \mathrm{~m}$ hurdles. From the grey relational sequence and weight, the biggest contribution to speed event-group is $400 \mathrm{~m}$, which reflects their training level of $400 \mathrm{~m}$ is relatively high, while $110 \mathrm{~m}$ hurdles is relatively weak.

(5)The grey relational sequence of individual event against speed event-group in the 21th to 23th stage is $110 \mathrm{~m}$ hurdles $>100 \mathrm{~m}>400 \mathrm{~m}$ From the grey relational sequence and weight, the biggest contribution to speed event-group is $110 \mathrm{~m}$ hurdles, which reflects their training level of $110 \mathrm{~m}$ hurdles is relatively high, while $400 \mathrm{~m}$ is relatively weak.

(6)The grey relational sequence of individual event against speed event-group in the 24th to 26th stage is $100 \mathrm{~m}>400 \mathrm{~m}>110 \mathrm{~m}$ hurdles. From the grey relational sequence and weight, the biggest contribution to speed event-group is $100 \mathrm{~m}$ hurdles, which reflects their training level of $100 \mathrm{~m}$ hurdles is relatively high, while $110 \mathrm{~m}$ hurdles is relatively weak.

(7)The grey relational sequence of individual event against speed event-group in the 27th to 30th stage is $100 \mathrm{~m}>400 \mathrm{~m}>110 \mathrm{~m}$ hurdles. From the grey relational sequence and weight, the biggest contribution to speed event-group is $100 \mathrm{~m}$ hurdles, which reflects their training level of $100 \mathrm{~m}$ hurdles is relatively high, while $110 \mathrm{~m}$ hurdles is relatively weak. 
From table III, (1)The grey relational sequence of individual event against skip event-group in the 7th to 9th stage is long jump $>$ pole vault $>$ high jump. From the grey relational sequence and weight, the biggest contribution to skip event-group is long jump, which reflects their training level of long jump is relatively high, while high jump is relatively weak.

(2)The grey relational sequence of individual event against skip event-group in the 10th to 14th stage is long jump>high jump>pole vault. From the grey relational sequence and weight, the biggest contribution to skip event-group is long jump, which reflects their training level of long jump is relatively high, while pole vault is relatively weak.

TABLE III. GREY CORRELATION DEGREE, RELATIONAL SEQUENCE, WEIGHT AND WEIGHT SEQUENCE OF SKIP EVENT-GROUP AND INDIVIDUAL EVENT IN DIFFERENT STAGES

\begin{tabular}{|c|c|c|c|c|}
\hline Period & References & $\begin{array}{c}\text { Long } \\
\text { jump }\end{array}$ & High jump & Pole vault \\
\hline \multirow{3}{*}{$7-9$} & GRD & 0.6851 & 0.4599 & 0.4955 \\
\cline { 2 - 5 } & GRS & 1 & 3 & 2 \\
\cline { 2 - 5 } & W(\%) & $41.76 \%$ & $28.04 \%$ & $30.20 \%$ \\
\hline \multirow{3}{*}{$10-14$} & GRD & 0.7571 & 0.4790 & 0.4667 \\
\cline { 2 - 5 } & GRS & 1 & 2 & 3 \\
\cline { 2 - 5 } & W(\%) & $44.47 \%$ & $28.13 \%$ & $27.41 \%$ \\
\hline \multirow{3}{*}{$15-17$} & GRD & 0.6971 & 0.6418 & 0.4452 \\
\cline { 2 - 5 } & GRS & 1 & 2 & 3 \\
\cline { 2 - 5 } & W(\%) & $39.07 \%$ & $35.97 \%$ & $24.95 \%$ \\
\hline \multirow{3}{*}{$21-23$} & GRD & 0.4706 & 0.4602 & 0.5567 \\
\cline { 2 - 5 } & GRS & 2 & 3 & 1 \\
\cline { 2 - 5 } & W(\%) & $31.64 \%$ & $30.94 \%$ & $37.42 \%$ \\
\cline { 2 - 5 } & GRD & 0.4645 & 0.5381 & 0.4915 \\
\hline \multirow{3}{*}{$24-26$} & GRS & 3 & 1 & 2 \\
\cline { 2 - 5 } & W(\%) & $31.09 \%$ & $36.01 \%$ & $32.90 \%$ \\
\cline { 2 - 5 } & GRD & 0.6954 & 0.4591 & 0.5674 \\
\hline \multirow{3}{*}{$27-30$} & W(\%) & $40.39 \%$ & $26.66 \%$ & $32.95 \%$ \\
\cline { 2 - 5 } & GRD & 0.7685 & 0.5403 & 0.6787 \\
\cline { 2 - 5 } & GRS & 1 & 3 & 2 \\
\hline \multirow{3}{*}{ W(\%) } & $38.67 \%$ & $27.18 \%$ & $34.15 \%$ \\
\hline
\end{tabular}

(3)The grey relational sequence of individual event against skip event-group in the 15th to 17th stage is long jump>high jump>pole vault. From the grey relational sequence and weight, the biggest contribution to skip event-group is long jump, which reflects their training level of long jump is relatively high, while pole vault is relatively weak.

(4)The grey relational sequence of individual event against skip event-group in the 18th to 20th stage is pole vault $>$ long jump>high jump. From the grey relational sequence and weight, the biggest contribution to skip event-group is pole vault, which reflects their training level of pole vault is relatively high, while high jump is relatively weak.

(5)The grey relational sequence of individual event against skip event-group in the 21th to 23th stage is high jump>pole vault $>$ long jump. From the grey relational sequence and weight, the biggest contribution to skip event-group is high jump, which reflects their training level of high jump is relatively high, while long jump is relatively weak.
(6)The grey relational sequence of individual event against skip event-group in the 24th to 26th stage is long jump>pole vault>high jump. From the grey relational sequence and weight, the biggest contribution to skip event-group is long jump, which reflects their training level of long jump is relatively high, while high jump is relatively weak.

(7)The grey relational sequence of individual event against skip event-group in the 27th to 30th stage is long jump>pole vault>high jump. From the grey relational sequence and weight, the biggest contribution to skip event-group is long jump, which reflects their training level of long jump is relatively high, while high jump is relatively weak.

TABLE IV. GREY CORRELATION DEGREE, RELATIONAL SEQUENCE, WEIGHT AND WEIGHT SEQUENCE OF THROWING EVENT-GROUP AND INDIVIDUAL EVENT IN DIFFERENT STAGES

\begin{tabular}{|c|c|c|c|c|}
\hline Period & References & Shot put & Discus & Javelin \\
\hline \multirow{3}{*}{$7-9$} & GRD & 0.4643 & 0.4833 & 0.7373 \\
\cline { 2 - 5 } & GRS & 3 & 2 & 1 \\
\cline { 2 - 5 } & W(\%) & $27.56 \%$ & $28.68 \%$ & $43.76 \%$ \\
\hline \multirow{3}{*}{$10-14$} & GRD & 0.6945 & 0.5191 & 0.5504 \\
\cline { 2 - 5 } & GRS & 1 & 3 & 2 \\
\cline { 2 - 5 } $15-17$ & W(\%) & $39.37 \%$ & $29.43 \%$ & $31.20 \%$ \\
\cline { 2 - 5 } & GRD & 0.8382 & 0.4960 & 0.4502 \\
\cline { 2 - 5 } $18-20$ & GRS & 1 & 2 & 3 \\
\hline & W(\%) & $46.98 \%$ & $27.80 \%$ & $25.23 \%$ \\
\cline { 2 - 5 } & GRD & 0.5083 & 0.6232 & 0.5440 \\
\hline \multirow{3}{*}{$21-23$} & W(\%) & $30.34 \%$ & $37.20 \%$ & $32.47 \%$ \\
\cline { 2 - 5 } & GRD & 0.4648 & 0.5378 & 0.7189 \\
\cline { 2 - 5 } & GRS & 3 & 2 & 1 \\
\hline \multirow{3}{*}{$24-26$} & W(\%) & $27.00 \%$ & $31.24 \%$ & $41.76 \%$ \\
\cline { 2 - 5 } & GRD & 0.6651 & 0.5613 & 0.5138 \\
\cline { 2 - 5 } & GRS & 1 & 2 & 3 \\
\hline \multirow{3}{*}{$27-30$} & W(\%) & $38.22 \%$ & $32.26 \%$ & $29.52 \%$ \\
\cline { 2 - 5 } & GRD & 0.6684 & 0.5497 & 0.7499 \\
\cline { 2 - 5 } & GRS & 2 & 3 & 1 \\
\hline
\end{tabular}

From table IV, (1)The grey relational sequence of individual event against throwing event-group in the 7th to 9th stage is javelin>discus > shot put. From the grey relational sequence and weight, the biggest contribution to throwing event-group is javelin, which reflects their training level of javelin is relatively high, while shot put is relatively weak.

(2)The grey relational sequence of individual event against throwing event-group in the 10th to 14th stage is shot put $>$ javelin $>$ discus. From the grey relational sequence and weight, the biggest contribution to throwing eventgroup is shot put, which reflects their training level of shot put is relatively high, while discus is relatively weak.

(3)The grey relational sequence of individual event against throwing event-group in the 15th to 17th stage is shot put $>$ discus $>$ javelin. From the grey relational sequence and weight, the biggest contribution to throwing eventgroup is shot put, which reflects their training level of shot put is relatively high, while javelin is relatively weak.

(4)The grey relational sequence of individual event against throwing event-group in the 18th to 20th stage is discus $>$ javelin $>$ shot put. From the grey relational sequence 
and weight, the biggest contribution to throwing eventgroup is discus, which reflects their training level of discus is relatively high, while shot put is relatively weak.

(5)The grey relational sequence of individual event against throwing event-group in the 21th to 23th stage is javelin $>$ discus $>$ shot put. From the grey relational sequence and weight, the biggest contribution to throwing eventgroup is javelin, which reflects their training level of javelin is relatively high, while shot put is relatively weak.

(6)The grey relational sequence of individual event against throwing event-group in the 24th to 26th stage is shot put $>$ discus $>$ javelin. From the grey relational sequence and weight, the biggest contribution to throwing eventgroup is shot put, which reflects their training level of shot put is relatively high, while javelin is relatively weak.

(7)The grey relational sequence of individual event against throwing event-group in the 27th to 30th stage is javelin $>$ shot put $>$ discus. From the grey relational sequence and weight, the biggest contribution to throwing event-group is javelin, which reflects their training level of javelin is relatively high, while discus is relatively weak.

\section{CONCONUSION}

1. Based on the grey correlation degree and sequence of decathlon athletes' four event-groups against total achievements of modern Olympic champion in each stage, the grey correlation degree and weight is fluctuated among different stages. The ranking of speed event-group is 2,2 , $4,2,1,3,2$; The ranking of skip event-group is $3,1,2,3,3$, 1,1 ; The ranking of throwing event-group is $3,4,3,1,2,2$, 3 ; and the ranking of endurance event-group is $1,4,1,4,4$, $4,4$.

2. Based on the grey correlation degree and sequence of decathlon athletes' individual event against event-group achievements of modern Olympic champion in each stage. The ranking of $100 \mathrm{~m}$ of speed event-group is $2,3,1,2,2$, 1,1 ; the ranking of $400 \mathrm{~m}$ is: $1,2,3,1,3,2,2$; the ranking of 110 meters hurdles is $3,1,2,3,1,3,3$. The ranking of long jump of skip event-group is $1,1,1,2,3,1,1$; the ranking of high jump is $3,2,2,3,1,3,3$, and the ranking of pole vault is $2,3,3,1,2,2,2$. The ranking of shot put of throwing event-group is $3,1,1,3,3,1,2$; the ranking of discus is $2,3,2,1,2,2,3$, and the ranking of javelin is 1,2 , $3,2,1,3,1$.
3. In our study, we concluded further that there are certain inner relations of total result and event-groups, and also shown in the event-group and each individual event during the different stages. There are training guidance and ability characteristics of champion athlete athletics respectively in each stage of the Olympic game. Athletes' competitive ability is not in accordance with the unified model structure, and is a kind of scale structure. The study finds that endurance event-group is a bottleneck of men's decathlon, and is a key limiting factors in breakthrough. We may be trapped in the endurance bottleneck project if there is no the right solution or direction to resolve it. How to improve the endurance event performance is the big problem for the coaches and athletes without affecting the other events. To win in Olympic competition or break through 9000 points, all we have to do is to balanced develop four factors: speed event-group, jumping eventgroup, throwing event-group and endurance event.

\section{ACKNOWLEDGMENT}

This work is supported by Hunan province teaching reform research project(G21209), Hunan province colleges and universities scientific research project(11C0545), and Hunan province department of education project (11C0546).

\section{REFERENCES}

[1] Deng Julong. “Grey System Theory Base (3rd Edition)” Hubei, China: huazhong university of science and technology press,2002,pp. 122-171.

[2] LIU Sifeng, Dang Yaoguo, FANG Zhijian ."Grey System Theory and Application(3rd Edition)”, Beijing, China: science press,2004,pp. 50-76.

[3] TIAN Mai-jiu, Theories of Sport Training, Higher education press, 2007:49-50.

[4] WEN Chao, Track and Field Sports Advanced Tutorial ,Beijing: people's sport publishing house, 2000:574-600.

[5] LU Gang,WANG Zong-ping. The Development of Man's Decathlon Performance of Top Three in Athens Olympic Games, China Sport Science and Technology, 2005,41(5):24-26.

[6] TIAN Zhongyuan, ZHAO Lunan,XU Qing, The Athletic Results Characteristics of the Excellent Decathlete in China and in the World, Journal of Tianjin University of Sport, 2009,24(1):66-68.

[7] ZHENG Yi-le; JIA Chang-zhi. Gray Correlative Analysis of the Achievement of Top Chinese and Foreign Decathlon Athletes, Journal of Beijing University of Physical Education,2004,24(8): 1135-1136. 